\title{
Effects of Particle-Dispersion on the Strength of an Alumina Fiber-Reinforced Aluminum Alloy Matrix Composite
}

\author{
Kazunori Asano and Hiroyuki Yoneda \\ Department of Mechanical Engineering, Faculty of Science and Engineering, Kinki University, Higashiosaka 577-8502, Japan
}

Aluminum alloy matrix hybrid composites, in which alumina particles were dispersed among continuous alumina fibers, were fabricated by squeeze casting, and the influence of temperature and the effects of the particle-dispersion on the strength of the composites were then investigated. The particle-dispersion among the fibers minimized preform contraction and fiber-to-fiber contact due to the melt infiltration during the squeeze casting. The tensile strength, $0.2 \%$ proof stress and elastic modulus of the composites in the longitudinal direction increased with increasing the fiber volume fraction, retaining nearly the same values up to $623 \mathrm{~K}$. These values of the hybrid composite were larger than those of the particle-free composite at every temperature. This is because the fibers were distributed uniformly owing to the particles that prevented the fiber-to-fiber contact, leading to the reduction of stress concentration at the points of direct fiber contact, and stress transmission between the fiber and the matrix becomes easy. At every temperature, the transverse tensile strength and proof stress of the particle-free composite was lower than that of the unreinforced alloy, because the fracture was initiated at the fiber-to-fiber contact point and the cracks propagated mainly along the fiber-matrix interface. In contrast, the strength of the hybrid composite was close to that of the unreinforced alloy even at high-temperature, because the cracks propagated mainly throughout the matrix owing to the uniform distribution of the fibers and the strong fiber-matrix bond.

(Received February 6, 2003; Accepted April 21, 2003)

Keywords: aluminum alloy, hybrid composite, continuous alumina fiber, alumina particle, squeeze casting, mechanical properties, fractography

\section{Introduction}

There are many reports on the properties of the continuous fiber-reinforced aluminum alloy matrix composites because the reinforcement with the fiber considerably improves the strength of the aluminum alloy not only at room temperature but also at high temperature. ${ }^{1-9)}$ These composites are mainly fabricated by squeeze casting ${ }^{1-7)}$ and hot pressing. ${ }^{8,9)}$ Squeeze casting is an attractive process because it can be used even poor wettability between the melt and reinforcement ${ }^{10)}$ and is suitable for strengthening selected regions of the casting and processing complex shapes. ${ }^{11)}$ Also, it shows high cost performance. ${ }^{12)}$ However, because of the fiber contact, attributed to the contraction of the preform during melt infiltration, the transverse mechanical properties obtained are not sufficient. To prevent the fiber contact, dispersion of fine particles or whiskers between the fibers in the composite using a preform of the fibers having particles or whiskers attached to their surfaces has been proposed. The composite is called a hybrid composite. Aluminum alloy matrix hybrid composites have been fabricated using the following reinforcements: continuous $\mathrm{SiC}$ fibers having $\mathrm{SiC}$ particles attached to their surfaces, ${ }^{13)}$ continuous carbon fibers having $\mathrm{SiC}$ particles and whiskers attached to their surfaces, ${ }^{14,15)}$ and continuous Tyranno fibers having $\beta$-SiC

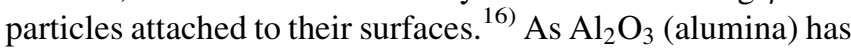
high rigidity and strength, and outstanding resistance to heat and wear, the alumina can improve the strength of aluminum alloy not only at room temperature but also at high temperature. With this improvement, the alloy could be used as structural heat-resistant parts. However, no reports on high-temperature properties of the hybrid composites using fine alumina particles and continuous alumina fibers could be found.

In this study, $\mathrm{Al}-\mathrm{Cu}-\mathrm{Mg}$ alloy matrix hybrid composites using continuous alumina fibers having fine alumina particles attached to their surfaces were produced by squeeze casting. The relationship among microstructure, strength characteristics in the range from room temperatute to $623 \mathrm{~K}$, and the volume fraction of the reinforcements examined. Additionally, the effects of the alumina particle-dispersion on the high-temperature strength and the strengthening mechanism were investigated based on fractography.

\section{Experimental Procedures}

JIS AC1B aluminum alloy $(\mathrm{Al}-4.3 \mathrm{Cu}-0.2 \mathrm{Mg}$ alloy) was used as the matrix metal, and continuous $\gamma$-alumina fibers (Sumitomo Chemical Inc., SV-10-1K) and $\alpha$-alumina particles (Showa Denko, Inc., CB-A01) were used as the reinforcement. The chemical composition of AC1B aluminum alloy and the characteristics of the reinforcements ${ }^{17)}$ are listed in Tables 1 and 2, respectively. It has been reported that the tensile strength of the fibers decreases little after a long-

Table 1 Chemical composition of AC1B (mass\%).

\begin{tabular}{ccccc}
\hline $\mathrm{Cu}$ & $\mathrm{Mg}$ & $\mathrm{Fe}$ & $\mathrm{Ti}$ & $\mathrm{Al}$ \\
\hline 4.31 & 0.20 & 0.15 & 0.14 & Bal. \\
\hline
\end{tabular}

Table 2 Properties of alumina fiber and alumina particle.

\begin{tabular}{lcc}
\hline & alumina fiber & alumina particle \\
\hline Diameter $(\mu \mathrm{m})$ & 15 & 1.0 \\
\hline Density $\left(\mathrm{Mg} / \mathrm{m}^{3}\right)$ & 3.3 & 4.0 \\
\hline Elastic modulus $(\mathrm{GPa})$ & 210 & - \\
\hline Tensile strength $(\mathrm{MPa})$ & 1800 & - \\
\hline Crystal phase & $\gamma$ & $\alpha$ \\
\hline Composition $($ mass $\%)$ & $\mathrm{Al}_{2} \mathrm{O}_{3} 85$ & $\mathrm{Al}_{2} \mathrm{O}_{3} 99.9$ \\
& $\mathrm{SiO}_{2} 15$ & \\
\hline
\end{tabular}



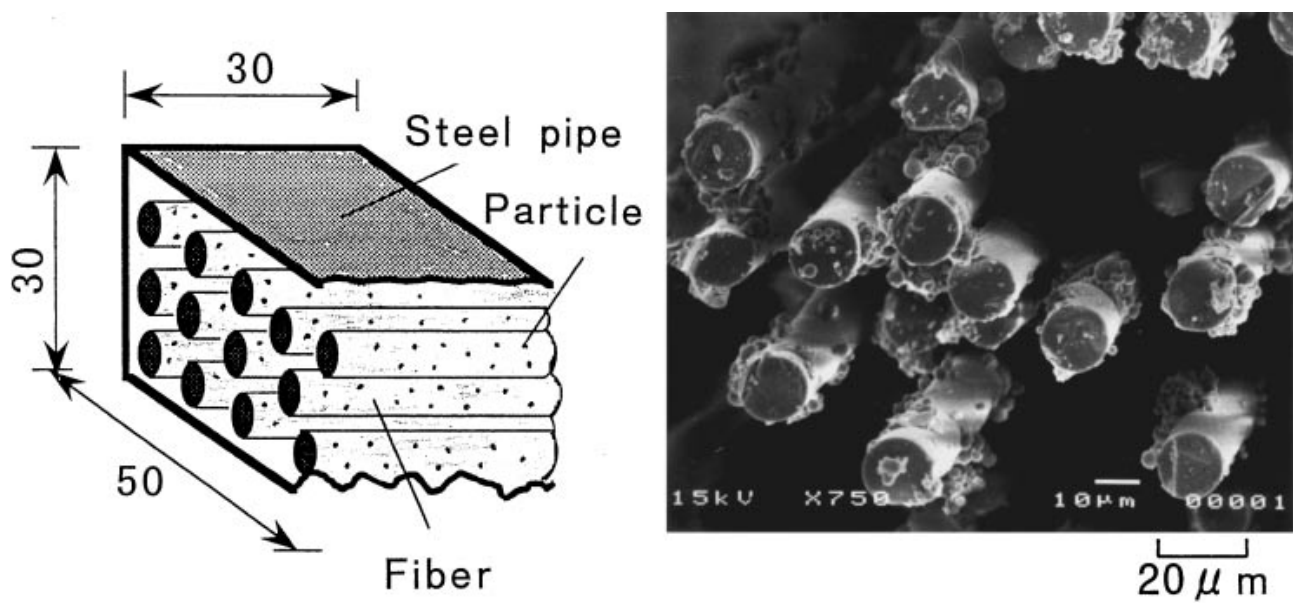

Fig. 1 Appearance of preform.

time exposure at $1373 \mathrm{~K}$ in the air. ${ }^{18)}$ The preform was fabricated as follows: (1) alumina particles were dispersed in water containing a small amount of polyvinyl alcohol as a binder, (2) the particles were attached to the fiber surfaces by soaking the fibers in the solution, and (3) the fibers were packed unidirectionally in a steel square pipe with open ends, dried at $373 \mathrm{~K}$, and then heated at $973 \mathrm{~K}$ for 1 hour to remove the binder in the preform. A schematic illustration and a SEM image of the preform are shown in Fig. 1. The particles can be seen attached to the fiber surfaces thus preventing fiber-tofiber contact.

The composite was fabricated by squeeze casting. The preform, still in the steel pipe, was placed horizontally in the mold, and the molten aluminum alloy was poured into the mold at $1073 \mathrm{~K}$. Pressure was quickly applied using a plunger and maintained until solidification was complete. The steel square pipe with open ends makes it easy to infiltrate the melt parallel to the fiber axis from both ends simultaneously. In this way, preform contraction is limited. The preform temperature was $973 \mathrm{~K}$, the mold temperature was $573 \mathrm{~K}$, the applied pressure was $40 \mathrm{MPa}$, and the pressurization retention time was $60 \mathrm{~s}$. The volume fraction of the fibers (hereinafter $V_{\mathrm{f}}$ ) was made 40,50 and $60 \%$, and the volume fraction of the particles (hereinafter $V_{\mathrm{p}}$ ) was 0 and $10 \%$. T6 heat treatment (solutionized at $788 \mathrm{~K}$ for $36 \mathrm{ks}$ and waterquenched, followed by aging at $433 \mathrm{~K}$ for $14.4 \mathrm{ks}$ ) was applied to the composites.

Their microstructures and the fiber-matrix interfaces were observed. Tensile tests in the longitudinal and transverse direction were conducted with machined plate specimens $3 \mathrm{~mm}$ in thickness, $8 \mathrm{~mm}$ in width and $10 \mathrm{~mm}$ in gage length to obtain tensile strength, $0.2 \%$ proof stress, elastic modulus and elongation in the range from room temperature $(293 \mathrm{~K})$ to $623 \mathrm{~K}$. In the tensile test, an Instron testing system was used and the crosshead speed was $1.67 \times 10^{-5} \mathrm{~m} / \mathrm{s}$. Specimen numbers were determined by the combination of $V_{\mathrm{f}}$ and $V_{\mathrm{p}}$. For example, composite $40-0$ contains $40 \%$ fibers and $0 \%$ particles, and composite $40-10$ contains $40 \%$ fibers and $10 \%$ particles.

\section{Results and Discussion}

\subsection{Microstructure}

Figure 2 shows the microstructures of the transverse section of particle-free composites (40-0 and 60-0) and
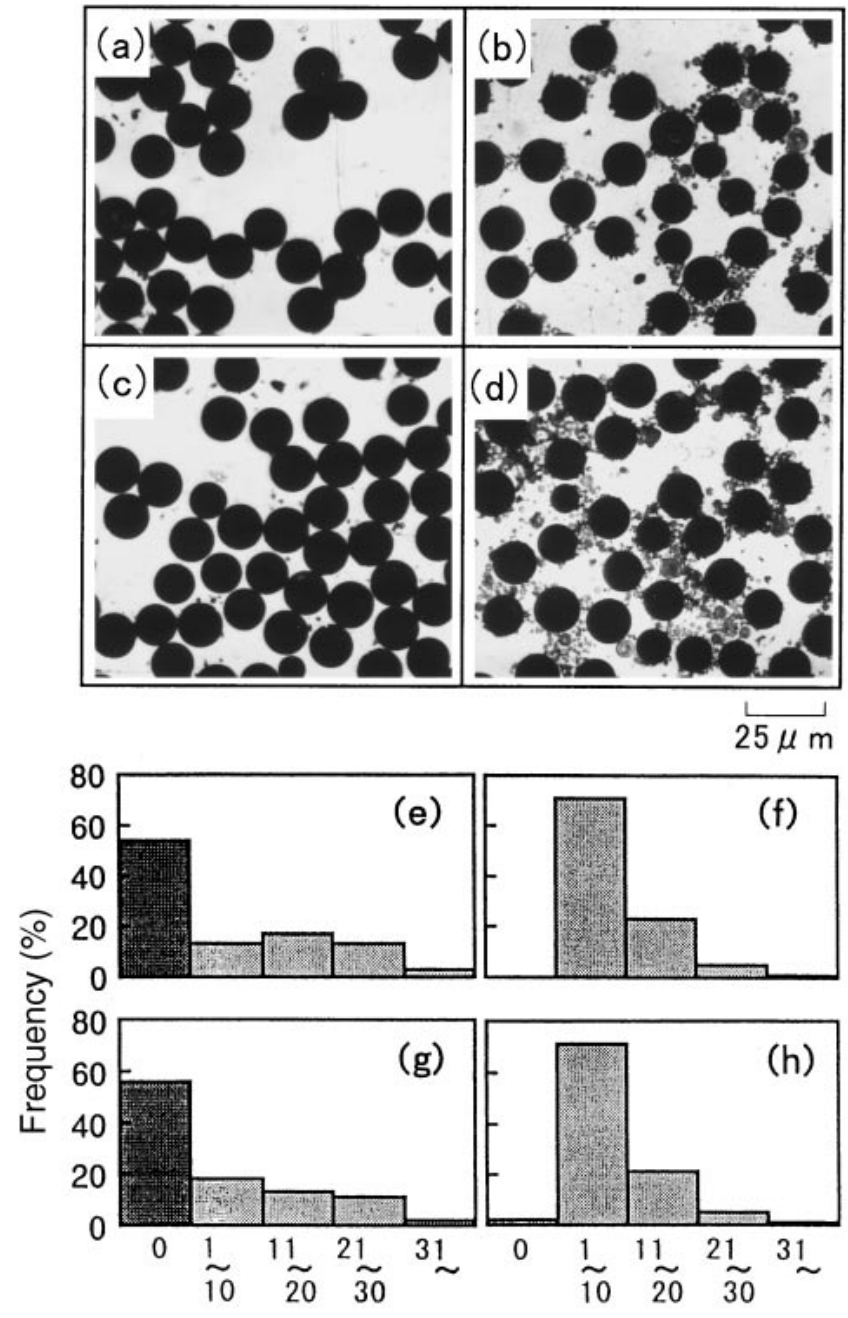

Distance between Adjacent Fibers, $d / \mu \mathrm{m}$

Fig. 2 Microstructures and fiber distribution in composites [(a) (e) 40-0, (b) (f) 40-10, (c) (g) 60-0, (d) (h) 60-10]. 


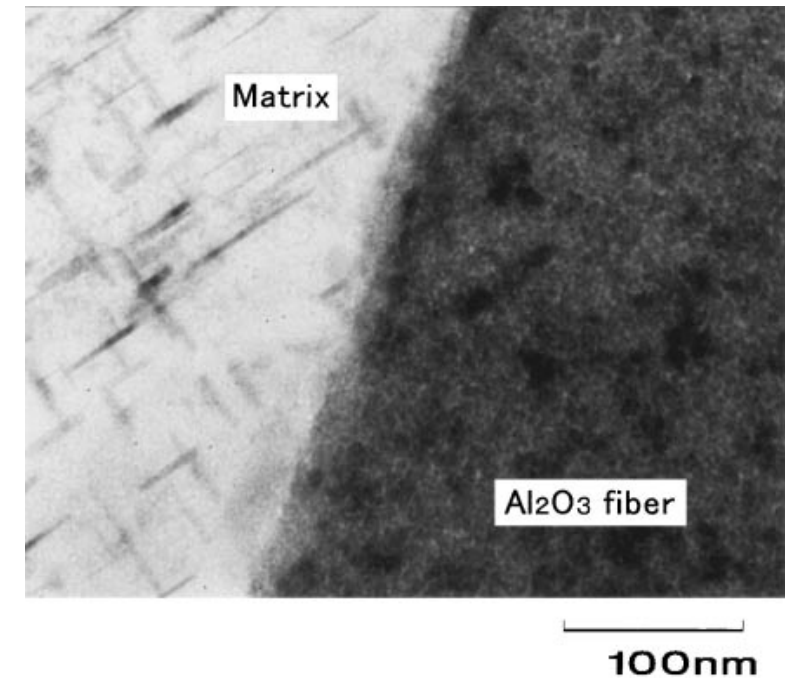

Fig. 3 TEM photograph of a region near a fiber-matrix interface in the composite (50-10).

hybrid composites (40-10 and 60-10). The frequency distributions of the distance between adjacent fibers are shown in Figs. 2(e) through (h). The distance between adjacent fibers was measured with an image analyzer using microstructure photographs. No defects or damage in the particles or fibers were observed in the microstructure. When the particles were not introduced, the fibers were distributed nonuniformly having fiber-to-fiber contact (Figs. 2(a) and (c)). The frequency of fibers touching was between $50 \%$ and $60 \%$ (Figs. 2(e) and (g)). In contrast, in the hybrid composites, the particles on the fiber surfaces prevented the fiber contact, and some particles were dispersed in the matrix (Figs. 2(b) and (d)). The frequency of fibers touching was almost nil, while that $1-10 \mu \mathrm{m}$ apart was about $70 \%$ (Figs. 2(f) and (h)). These results indicate that the fibers are distributed more uniformly in the hybrid composite.

A TEM image near the fiber-matrix interface in a hybrid composite (50-10) is shown in Fig. 3. There was no reaction product near the interface between the fiber and the matrix, indicating that the fibers bonded with the matrix without causing any excessive reaction or degradation. The grain size of the $\gamma$-alumina in the fiber is about $10 \mathrm{~nm}$, which is almost equal to the grain size in the as-received fiber. ${ }^{19)}$ This indicates that degradation of the fiber strength due to the grain growth during the de-binder process, the infiltration of molten aluminum alloy, and the solution treatment did not occur.

\subsection{Mechanical properties}

Figure 4 shows stress-strain curves of $50 \%$ fiber-reinforced composites tested at room temperature $(293 \mathrm{~K})$ and $623 \mathrm{~K}$. In the longitudinal direction, stress increased almost linearly as the strain increased at every temperature. Tensile resistance to deformation of the hybrid composite (50-10) was larger than that of the particle-free composite (50-0). Tensile resistance to deformation in the transverse direction was smaller than that in the longitudinal direction and decreased as the temperature increased. The resistance increased with the introduction of the particles as well as in the longitudinal direction.

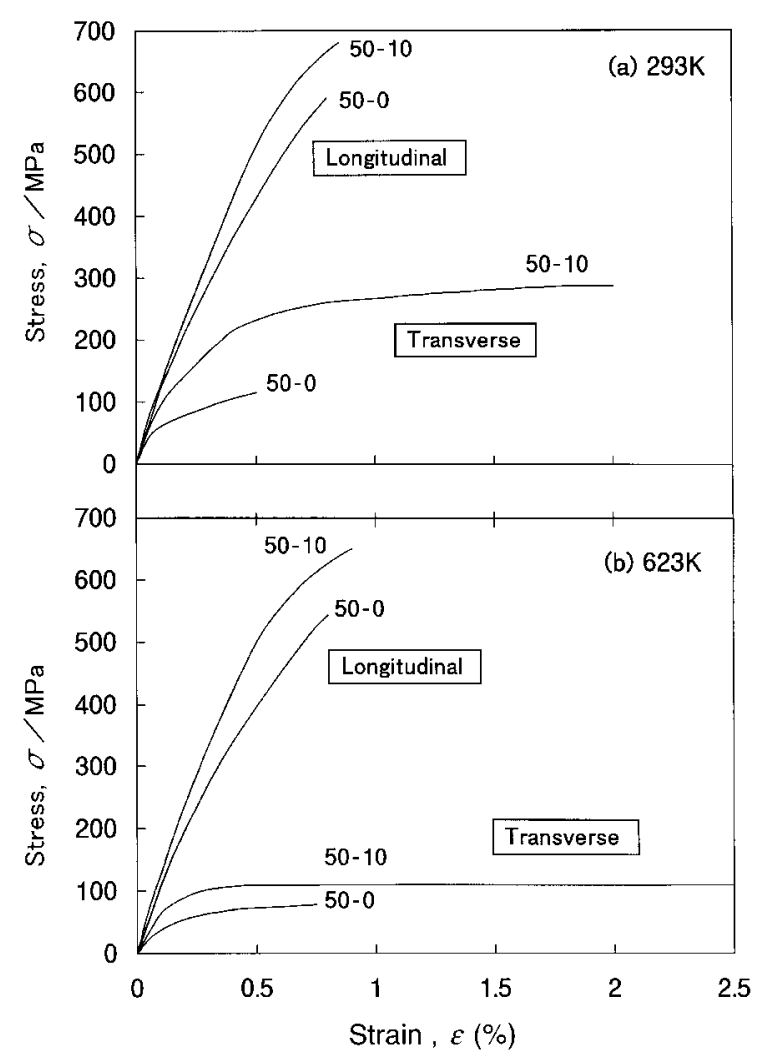

Fig. 4 Stress-strain curves of composites $\left(V_{\mathrm{f}}=50 \%\right)$ tested at (a) $293 \mathrm{~K}$ and (b) $623 \mathrm{~K}$.

Figure 5 shows the relationship between the tensile strength and the temperature for the unreinforced alloy and the composites. The tensile strength of the unreinforced alloy decreased little up to about $423 \mathrm{~K}$, above which it decreased considerably (indicated by the open circles in Fig. 5). For the composites, the longitudinal tensile strength increased as $V_{\mathrm{f}}$ increased and decreased little even at high-temperature. This is probably because the fibers are subject to longitudinal tensile stress. The longitudinal tensile strength of the hybrid composite was larger than that of the particle-free composite with the same $V_{\mathrm{f}}$. The transverse tensile strength of the particle-free composite were lower than those of the unreinforced alloy and decreased as $V_{\mathrm{f}}$ increased. They were also improved by the introduction of the particles; the transverse tensile strength of the hybrid composite were almost the same as those of the unreinforced alloy (Fig. 5(b)). The increase in the strength by the introduction of particles was particularly large up to about $423 \mathrm{~K}$, above which the increase was small owing to the low strength of the matrix. Nevertheless, the strength of the hybrid composite was slightly larger than that of the particle-free composite at $623 \mathrm{~K}$

Generally, the longitudinal tensile strength of the continuous fiber-reinforced composite can be estimated by the rule of mixture expressed as eq. (1). ${ }^{20)}$

$$
\sigma_{\text {rom }}=\sigma_{\mathrm{f}} V_{\mathrm{f}}+\sigma_{\mathrm{m}}^{\prime}\left(1-V_{\mathrm{f}}\right)
$$

where $\sigma_{\text {rom }}$ is the tensile strength of the composite, $\sigma_{\mathrm{f}}$ is the tensile strength of the fiber, and $\sigma_{\mathrm{m}}^{\prime}$ is the stress on the matrix when the fibers reach their ultimate tensile stress in the composite. Table 3 shows $\sigma_{\text {rom }}$, experimental values of the 


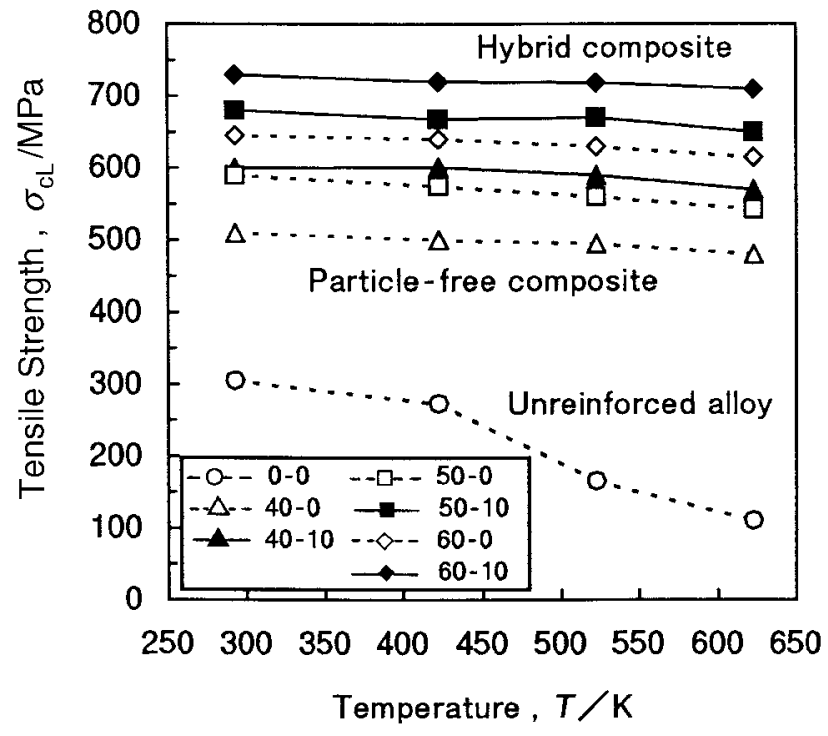

(a) Longitudinal direction

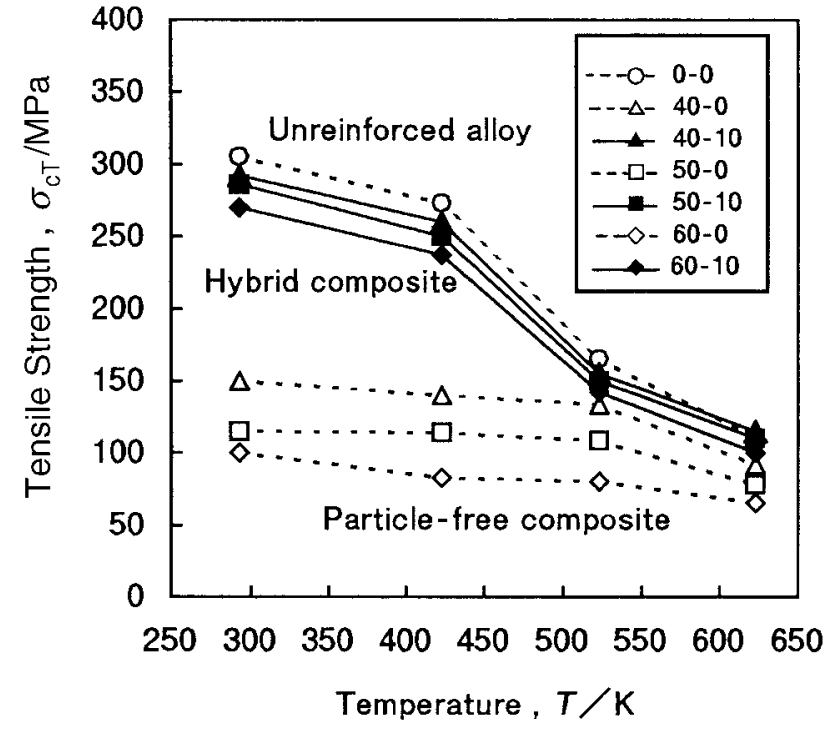

(b) Transverse direction

Fig. 5 Tensile strength of unreinforced alloy and composites at various temperatures.

Table 3 Comparison between experimental and calculated tensile strengths in the longitudinal direction.

\begin{tabular}{cccccccc}
\hline \multirow{2}{*}{ Specimen } & \multicolumn{4}{c}{$293 \mathrm{~K}$} & & \multicolumn{3}{c}{$623 \mathrm{~K}$} \\
\cline { 2 - 4 } \cline { 6 - 8 } & $\sigma_{\mathrm{cL}}$ & $\sigma_{\text {rom }}$ & $\sigma_{\mathrm{cL}} / \sigma_{\text {rom }}$ & & $\sigma_{\mathrm{cL}}$ & $\sigma_{\text {rom }}$ & $\sigma_{\mathrm{cL}} / \sigma_{\text {rom }}$ \\
\hline $40-0$ & 510 & 887 & 0.57 & & 480 & 782 & 0.61 \\
$40-10$ & 600 & 890 & 0.67 & & 570 & 782 & 0.73 \\
\hline $60-0$ & 645 & 1186 & 0.54 & & 615 & 1120 & 0.55 \\
$60-10$ & 730 & 1190 & 0.61 & & 710 & 1120 & 0.63 \\
\hline
\end{tabular}

$\sigma_{\mathrm{cL}}:$ Experimental tensile strength (MPa)

$\sigma_{\text {rom }}$ : Tensile strength calculated by rule of mixture (MPa)

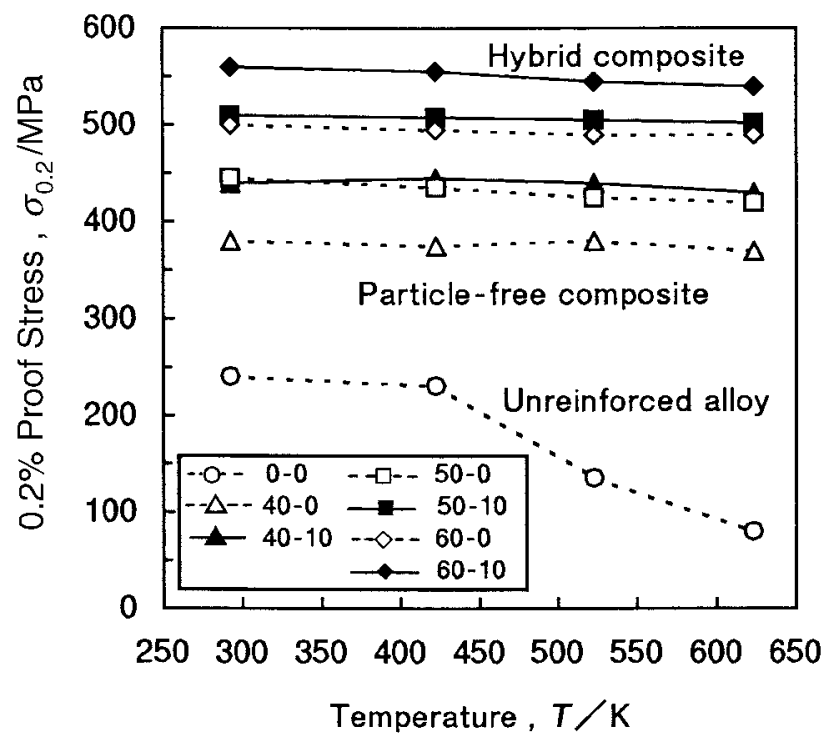

(a) Longitudinal direction tensile strength $\left(\sigma_{\mathrm{cL}}\right)$, and the ratios of $\sigma_{\mathrm{cL}}$ to $\sigma_{\mathrm{rom}}$. From Table 2 , the value of $\sigma_{\mathrm{f}}$ was set at $1800 \mathrm{MPa}$. Although $\sigma_{\mathrm{cL}}$ was smaller than $\sigma_{\text {rom }}$ for every composite, the tensile strength and the ratios $\sigma_{\mathrm{cL}} / \sigma_{\text {rom }}$ of the hybrid composites (4010 and 60-10) were larger than those of the particle-free composites. This suggests that the strengthening effect of the fibers with the particles is large.

Figure 6 shows the relationship between $0.2 \%$ proof stress and temperature for the unreinforced alloy and the composites. The variation in the $0.2 \%$ proof stress as a function of temperature was similar to that in the tensile strength in the longitudinal and transverse directions.

Figure 7 shows the relationship between the elastic modulus and the temperature for the unreinforced alloy and

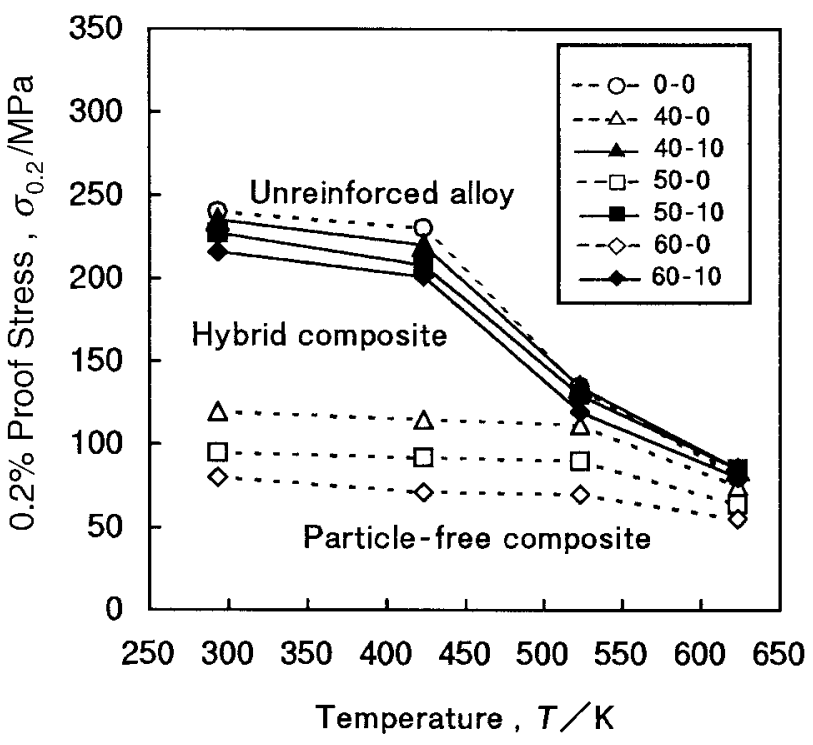

(b) Transverse direction

Fig. $6 \quad 0.2 \%$ proof stress of unreinforced alloy and composites at various temperatures. 


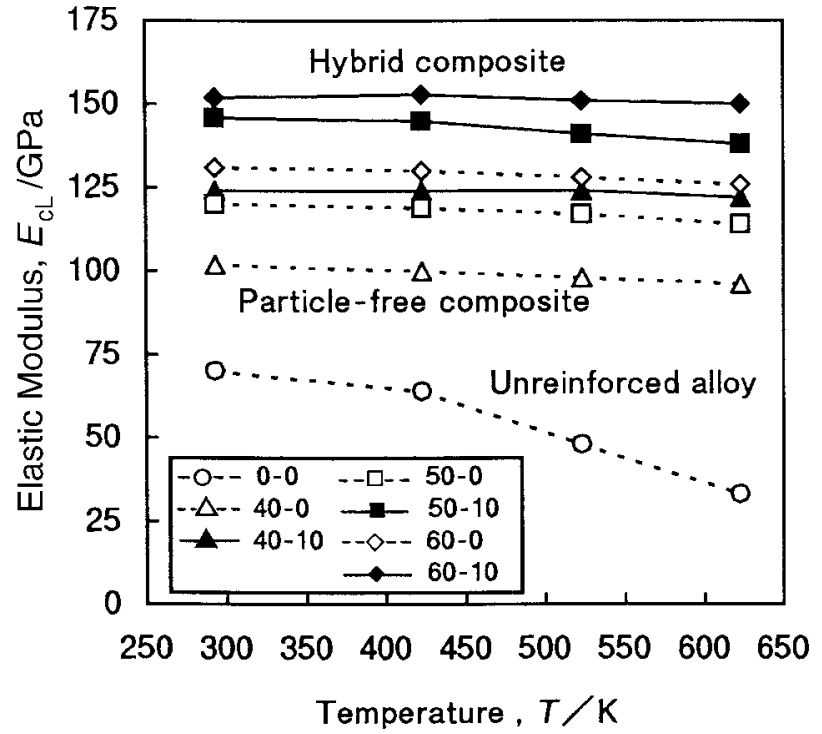

(a) Longitudinal direction

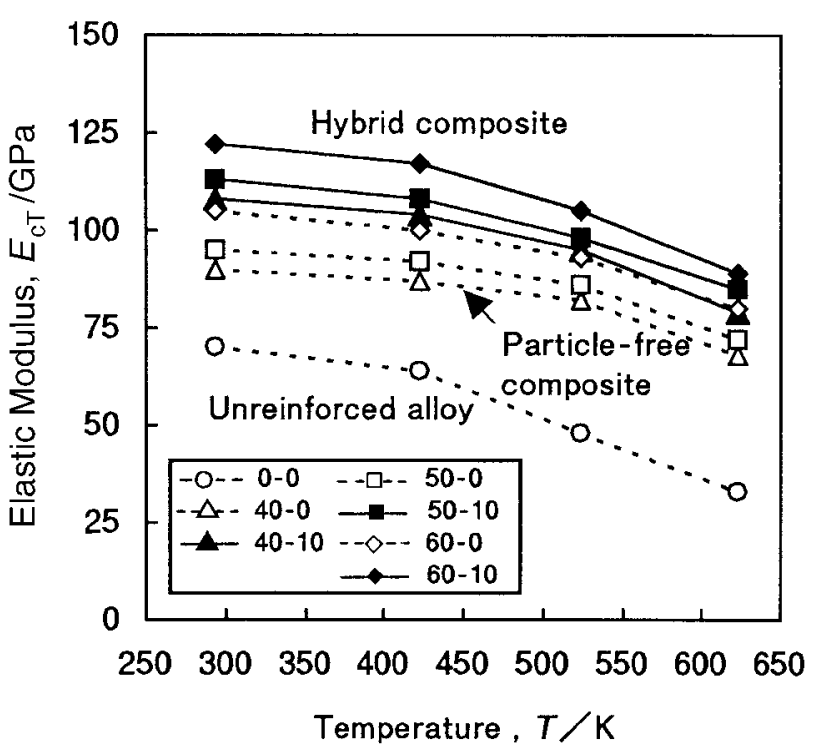

(b) Transverse direction

Fig. 7 Elastic modulus of unreinforced alloy and composites at various temperatures.

the composites. The longitudinal and transverse elastic modulus increased as $V_{\mathrm{f}}$ increased and further increased with the introduction of the particles. Although the longitudinal elastic modulus was almost constant over the temperature range measured, the transverse elastic modulus decreased as the temperature increased. The decrease in the transverse elastic modulus is probably because the elastic modulus of the matrix decreased as the temperature increased.

As well as the tensile strength, the longitudinal elastic modulus can be estimated by the rule of mixture expressed as eq. (2). ${ }^{20)}$

$$
E_{\mathrm{rom}}=E_{\mathrm{f}} V_{\mathrm{f}}+E_{\mathrm{m}}\left(1-V_{\mathrm{f}}\right)
$$

where $E_{\mathrm{rom}}, E_{\mathrm{f}}$ and $E_{\mathrm{m}}$ are the elastic modulus of the composite, the fiber and the matrix, respectively. From Table $2, E_{\mathrm{f}}$ was set at $210 \mathrm{GPa}$. Table 4 shows $E_{\text {rom }}$, the experimental elastic modulus $\left(E_{\mathrm{cL}}\right)$, and the ratios of $E_{\mathrm{cL}}$ to $E_{\mathrm{rom}}$. For the particle-free composite, $E_{\mathrm{cL}}$ was smaller than $E_{\text {rom. }}$. The introduction of the particles increased $E_{\mathrm{cL}}$, resulting in almost the same values as $E_{\text {rom }}$ at room temperature and slightly larger than $E_{\text {rom }}$ at $623 \mathrm{~K}$. This is probably because the introduction of the particles reduces the fiber-to-fiber contact and the stress transmission between the fiber and the matrix becomes easy, and increases the elastic

Table 4 Comparison between experimental and calculated elastic modulus in the longitudinal direction.

\begin{tabular}{cccccccc}
\hline \multirow{2}{*}{ Specimen } & \multicolumn{4}{c}{$293 \mathrm{~K}$} & & \multicolumn{3}{c}{$623 \mathrm{~K}$} \\
\cline { 2 - 4 } \cline { 6 - 8 } & $E_{\mathrm{cL}}$ & $E_{\mathrm{rom}}$ & $E_{\mathrm{cL}} / E_{\mathrm{rom}}$ & & $E_{\mathrm{cL}}$ & $E_{\mathrm{rom}}$ & $E_{\mathrm{cL}} / E_{\mathrm{rom}}$ \\
\hline $40-0$ & 102 & 126 & 0.81 & & 96 & 104 & 0.92 \\
$40-10$ & 124 & 126 & 0.98 & & 122 & 104 & 1.17 \\
\hline $60-0$ & 131 & 154 & 0.85 & & 126 & 139 & 0.91 \\
$60-10$ & 152 & 154 & 0.99 & & 150 & 139 & 1.08 \\
\hline
\end{tabular}

$E_{\mathrm{cL}}$ : Experimental elastic modulus $(\mathrm{GPa})$

$E_{\text {rom }}$ : Elastic modulus calculated by rule of mixture (GPa) modulus of the matrix $\left(E_{\mathrm{m}}\right)$.

Figure 8 shows the relationship between the elongation and the temperature for the unreinforced alloy and the composites. Although the elongation of the unreinforced alloy increased as the temperature increased, that of the particle-free composite in the longitudinal (Fig. 8(a)) and transverse (Fig. 8(b)) directions changed little even as the temperature increased. In contrast, the elongation of the hybrid composite increased as the temperature increased. This tendency was pronounced when the volume fraction of the matrix was large ( $V_{\mathrm{f}}$ was small).

\subsection{Relationship between strength and fracture mor- phology}

\subsubsection{Longitudinal fracture morphology}

Figure 9 shows the fracture surfaces of the composites after a longitudinal tensile load was applied at room temperature. There is almost no fiber pullout on either fracture surface, suggesting that the interfacial bond between the fibers and the matrix was relatively strong. Under more specific observation, the surface of the particle-free composite was flat at the fiber-to-fiber contact points (arrows in the Fig. 9(a-1)). Fig. 9(a-2) is a high magnification micrograph near the contact point, showing the chevron patterns on the fracture surfaces of the fibers initiated at the fiber-to-fiber contact points indicated by arrows. In contrast, the surface of the hybrid composite was irregular and a flat region was not observed (Fig. 9(b-1)). The chevron patterns were observed on the fracture surfaces of the fibers, but the initial points of the fracture varied: the surface of the fiber, the interior of the fiber (Fig. 9(b-2)).

Figure 10 shows the fracture surfaces of the composites at $623 \mathrm{~K}$. The variation in the morphology of the fracture surface by the particle-dispersion was similar to that at room temperature.

Based on these fractographs, the fracture morphology when the tensile load was applied in the longitudinal 


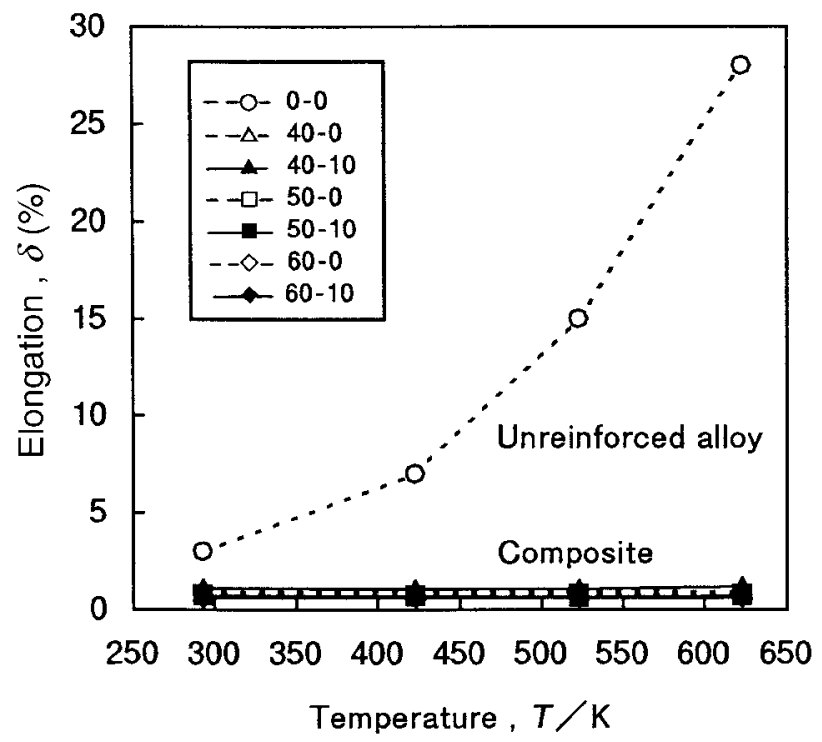

(a) Longitudinal direction

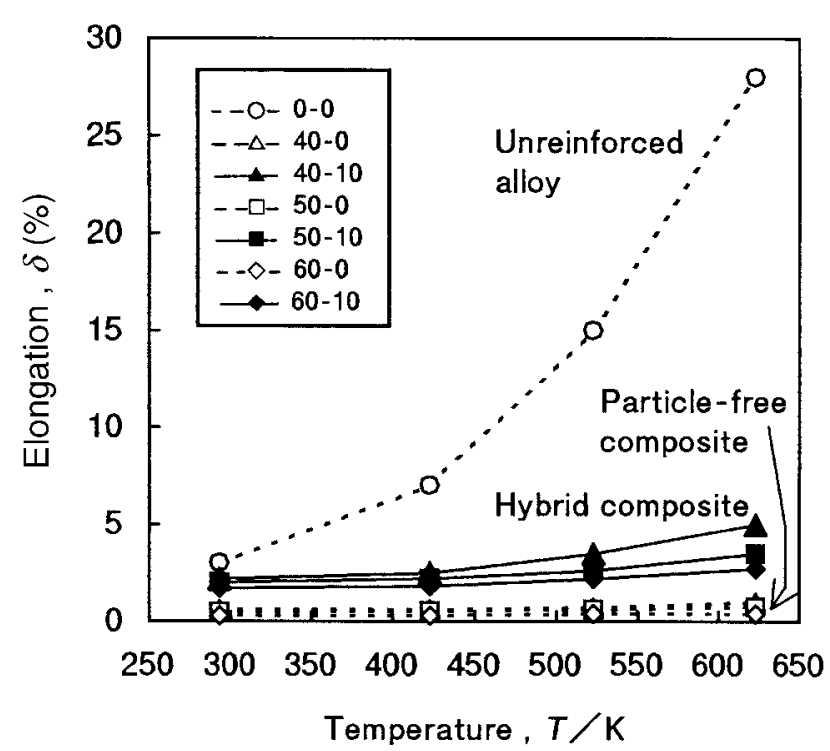

(b) Transverse direction

Fig. 8 Elongation of unreinforced alloy and composites at various temperatures.

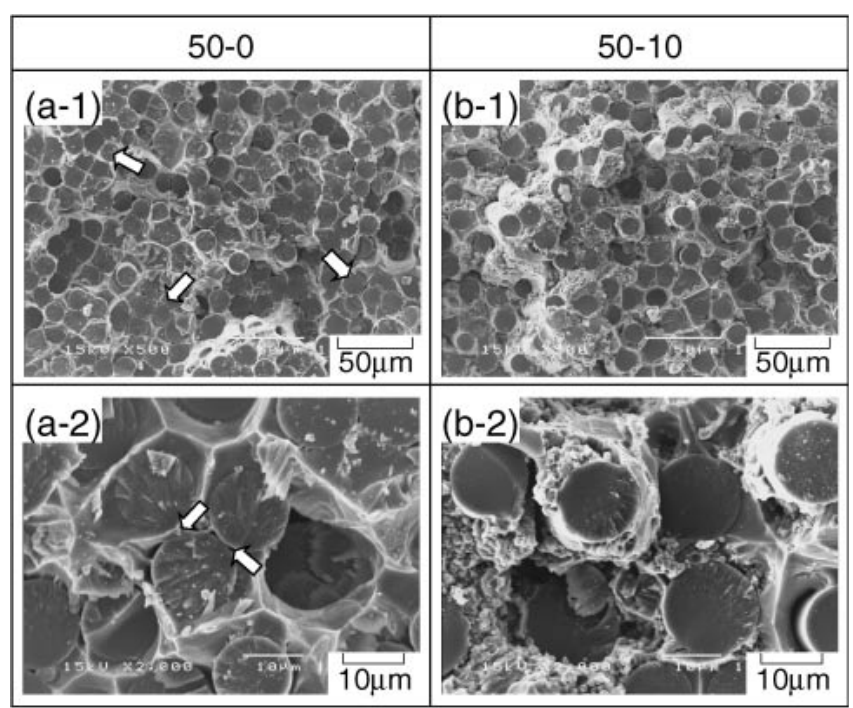

Fig. 9 SEM photographs of longitudinal fracture surfaces of composites after tensile test at $293 \mathrm{~K}$.

direction was considered. The model for the crack propagation is shown in Fig. 11. When a fiber breaks, a neighboring fiber directly contacted by the broken fiber breaks easily because stress concentration occurs in the neighboring fiber. Close-packed fibers were frequently observed on the fracture surfaces of the particle-free composite. Thus, progressive fracture of touching fibers was considered to occur easily, as shown in Fig. 11(a). When the touching fibers break, the fracture surface must be flat. This would be why the surface of the particle-free composite became flat. Under this fracture morphology, the strengthening effect of the fiber cannot be fully displayed. In contrast, for the hybrid composite, in which the fibers were distributed uniformly, the matrix around a fiber relieves the stress concentration after the fiber breaks. Thus the stress concentration does not easily occur,

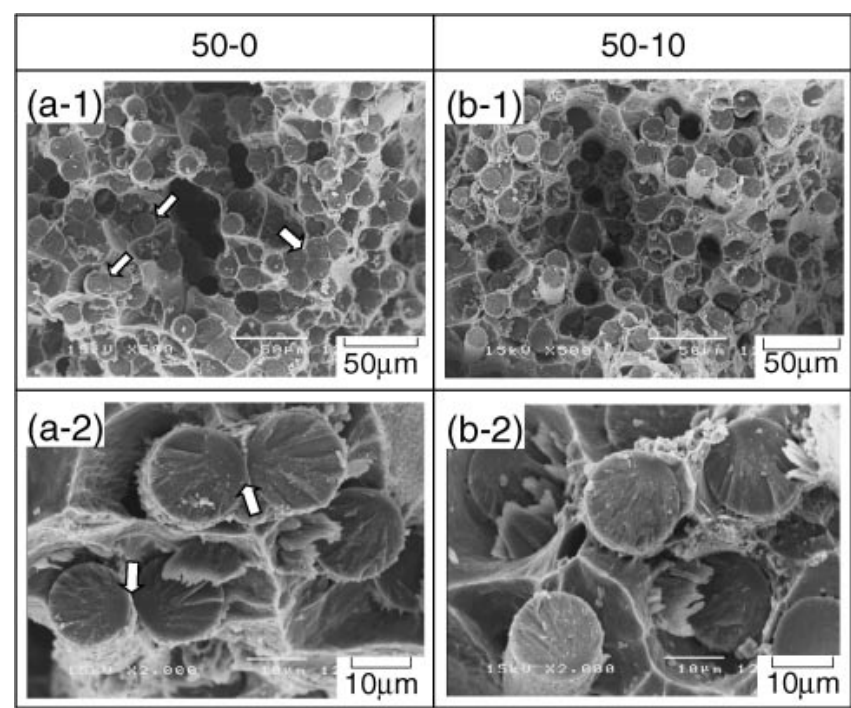

Fig. 10 SEM photographs of longitudinal fracture surfaces of composites after tensile test at $623 \mathrm{~K}$.

and each fiber is subject to the stress. Consequently, irregular surfaces are frequently formed as shown in Fig. 11(b). That is, because the introduction of the particles reduces the fiberto-fiber contact and the stress transmission between the fiber and the matrix becomes easy, the longitudinal strength of the hybrid composite was higher than that of the particle-free composite. At higher temperature, stress relaxation by the matrix would be more pronounced because the ductility of the matrix increased. This idea is supported by the fact that the ratios $\sigma_{\mathrm{cL}} / \sigma_{\text {rom }}$ at $623 \mathrm{~K}$ are larger than those at room temperature with the same $V_{\mathrm{f}}$, as shown in Table 3 .

\subsubsection{Transverse fracture morphology}

Figure 12 shows the fracture surfaces of the composites after a transverse tensile load was applied at room temperature. In this investigation, both sides of the fracture surfaces 


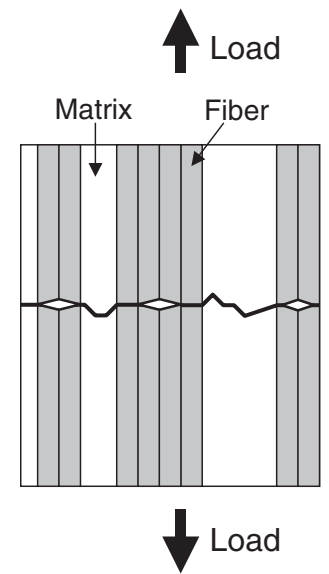

(a) Particle-free composite

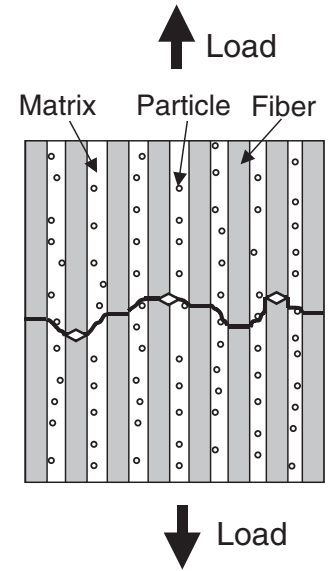

(b) Hybrid composite
Fig. 11 Schematic illustrations of the longitudinal fracture in composites.
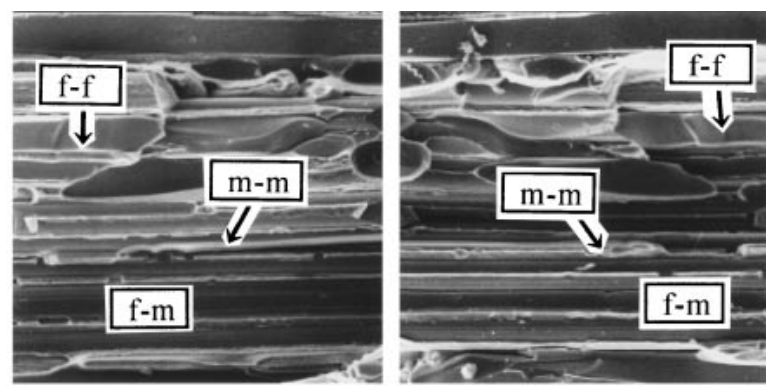

(a) Particle-free composite (50-0)

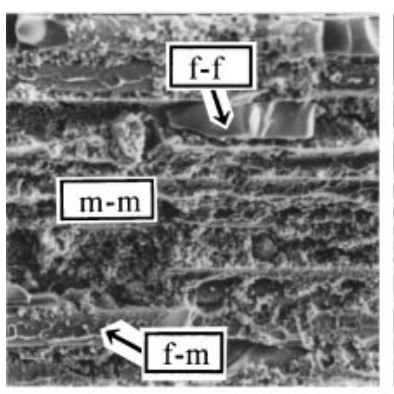

(b) Hybrid composite (50-10)

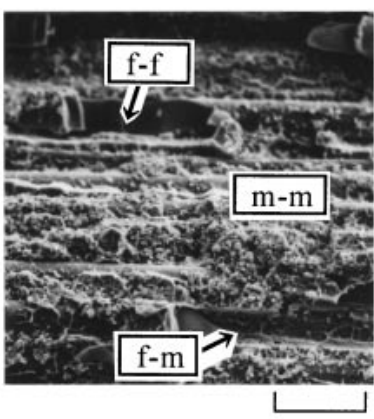

$25 \mu \mathrm{m}$
Fig. 12 SEM photographs of transverse fracture surfaces of composites after tensile test at $293 \mathrm{~K}$ (f-f: Fracture at fiber, f-m: Fracture at fibermatrix interface, $\mathrm{m}-\mathrm{m}$ : Fracture at matrix).

were observed to determine where the fracture occurred. On the fracture surface of the particle-free composite, many fibers and grooves remaining in the matrix can clearly be observed (Fig. 12(a)). In contrast, the matrix is dominant on the fracture surface of the hybrid composite (Fig. 12(b)). This suggests that the cracks propagated mainly throughout the matrix.

Figure 13 shows the fracture surfaces of the composites at $623 \mathrm{~K}$. The variation in the morphology of fracture surface by the particle-dispersion was similar to that at room temperature.

Based on these fractographs, the fracture morphology
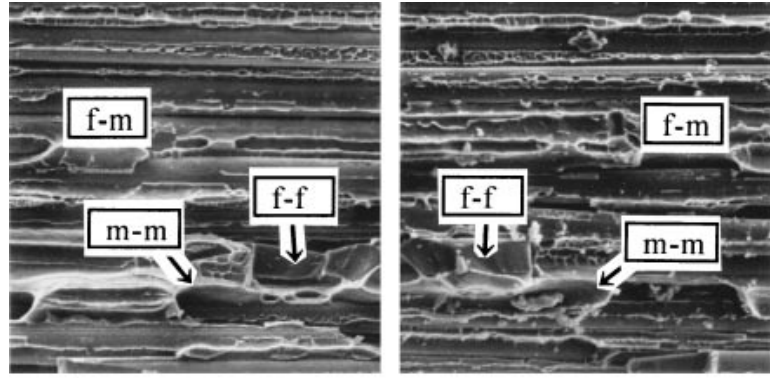

(a) Particle-free composite (50-0)
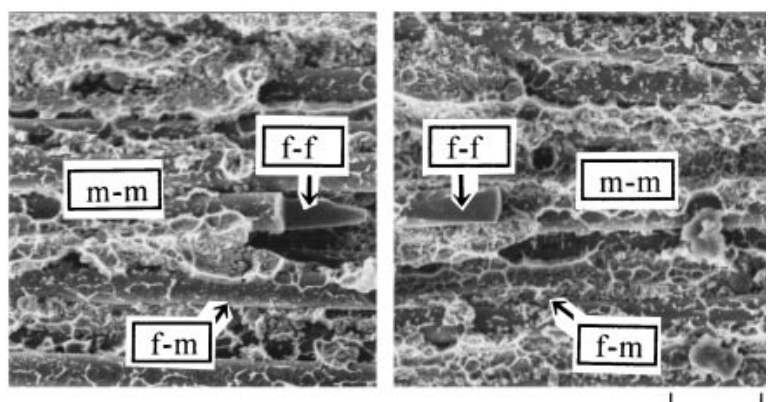

(b) Hybrid composite (50-10)

$25 \mu \mathrm{m}$

Fig. 13 SEM photographs of transverse fracture surfaces of composites after tensile test at $623 \mathrm{~K}$ (f-f: Fracture at fiber, f-m: Fracture at fibermatrix interface, m-m: Fracture at matrix).

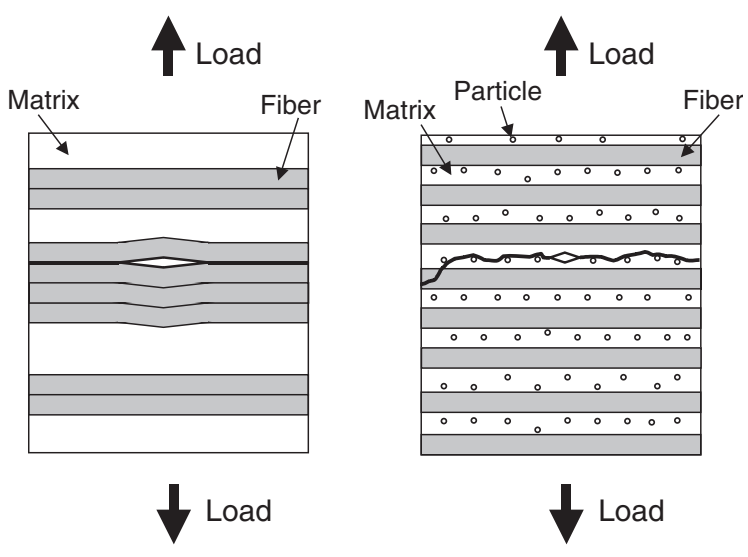

(a) Particle-free composite

(b) Hybrid composite

Fig. 14 Schematic illustrations of the transverse fracture in composites.

when the tensile load was applied in the transverse direction was considered. The model for crack propagation is shown in Fig. 14. The transverse tensile strength and elongation of the particle-free composite were significantly smaller than those of the unreinforced alloy. The fiber surfaces and grooves remaining in the matrix shown in Figs. 12 and 13 correspond to the points of direct fiber contact where the bond between the fibers and the matrix was insufficient. Because the stress transmission does not completely occur at the point of direct fiber contact, the cracks are considered to propagate selectively near the fiber-matrix interface, as shown in Fig. 14(a). The transverse tensile strength of the hybrid composite was close to that of the unreinforced alloy. This is probably 
Table 5 Transverse tensile strength of composites and each area fraction on the fracture surfaces.

\begin{tabular}{|c|c|c|c|c|c|c|c|c|}
\hline \multirow{2}{*}{ Specimen } & \multicolumn{4}{|c|}{$293 \mathrm{~K}$} & \multicolumn{4}{|c|}{$623 \mathrm{~K}$} \\
\hline & $A_{\mathrm{ff}}$ & $A_{\mathrm{fm}}$ & $A_{\mathrm{mm}}$ & $\sigma_{\mathrm{cT}}$ & $A_{\mathrm{ff}}$ & $A_{\mathrm{fm}}$ & $A_{\mathrm{mm}}$ & $\sigma_{\mathrm{cT}}$ \\
\hline $40-0$ & 13 & 47 & 40 & 150 & 4 & 51 & 45 & 92 \\
\hline $40-10$ & 12 & 9 & 79 & 290 & 4 & 23 & 73 & 116 \\
\hline $50-0$ & 14 & 65 & 21 & 115 & 6 & 70 & 24 & 78 \\
\hline $50-10$ & 10 & 17 & 73 & 288 & 5 & 33 & 62 & 110 \\
\hline $60-0$ & 15 & 77 & 8 & 100 & 7 & 76 & 17 & 65 \\
\hline $60-10$ & 10 & 31 & 59 & 260 & 5 & 46 & 49 & 101 \\
\hline
\end{tabular}

$A_{\mathrm{ff}}$ : Area fraction of fiber (\%)

$A_{\text {fm }}$ : Area fraction of fiber-matrix interface (\%)

$A_{\mathrm{mm}}$ : Area fraction of matrix (\%)

$\sigma_{\mathrm{cT}}$ : Transverse tensile strength $(\mathrm{MPa})$

due to the particles that distribute the fibers uniformly and prevent the fiber contact. Additionally, the interfacial bond between the fiber and the matrix was sufficient and strong. Consequently, the cracks are considered to propagate mainly throughout the matrix, as shown in Fig. 14(b).

In order to quantitatively analyze these fracture morphologies, the fracture surface was classified into three parts: the interface between fiber and matrix (f-m), matrix ( $m-m)$, and fiber (f-f). Each area fraction on the fracture surface was calculated, and the relationship between the area fraction and the transverse tensile strength was investigated. The results are summarized in Table 5. The area fraction of the interface, matrix, and fiber are expressed as $A_{\mathrm{fm}}, A_{\mathrm{mm}}$, and $A_{\mathrm{ff}}$, respectively. From Table 5, the following results were obtained: (1) $A_{\mathrm{ff}}$ is nearly constant at the same temperature, (2) $A_{\mathrm{fm}}$ increased as $V_{\mathrm{f}}$ increased, (3) $A_{\mathrm{fm}}$ was larger than $A_{\mathrm{mm}}$ in the particle-free composites, but smaller in the hybrid composites, and (4) $A_{\mathrm{mm}}$ of the hybrid composite was larger than that of the particle-free composite with the same $V_{\mathrm{f}}$. These results indicate that $A_{\mathrm{fm}}$ and $A_{\mathrm{mm}}$ have an appreciable influence on the transverse strength. The relationship between transverse tensile strength $\sigma_{\mathrm{cT}}(\mathrm{MPa}), A_{\mathrm{fm}}(\%)$, $A_{\mathrm{mm}}(\%)$, and $A_{\mathrm{ff}}(\%)$ was calculated by multiple regression analysis. As a result, the following eq. (3) was obtained at room temperature:

$$
\sigma_{\mathrm{cT}}=4.41 A_{\mathrm{mm}}+1.85 A_{\mathrm{fm}}-6.28 A_{\mathrm{ff}}
$$

The multiple correlation coefficient $(R)$ of eq. (3) is 0.978 , signifying substantial correlation. The relationship between each fraction can be expressed as

$$
A_{\mathrm{ff}}=100-\left(A_{\mathrm{mm}}+A_{\mathrm{fm}}\right)
$$

When eq. (4) is substituted in eq. (3), the following eq. (5) is obtained:

$$
\sigma_{\mathrm{cT}}=10.7 A_{\mathrm{mm}}+8.13 A_{\mathrm{fm}}-628
$$

$\sigma_{\text {cT }}$ of the composite $50-10$ calculated by eq. (5) is $291 \mathrm{MPa}$, and it is in good agreement with the experimental tensile strength of $288 \mathrm{MPa}$.

Likewise, the following eq. (6) was obtained at $623 \mathrm{~K}$ :

$$
\sigma_{\mathrm{cT}}=1.43 A_{\mathrm{mm}}+0.54 A_{\mathrm{fm}}-0.53 A_{\mathrm{ff}}
$$

$R$ of eq. (6) is 0.978 , signifying substantial correlation. When eq. (4) is substituted in eq. (6), the following equation is obtained:

$$
\sigma_{\mathrm{cT}}=1.96 A_{\mathrm{mm}}+1.07 A_{\mathrm{fm}}-53
$$

$\sigma_{\mathrm{cT}}$ of the composite 50-10 calculated by eq. (5) is $104 \mathrm{MPa}$ and is in good agreement with the experimental tensile strength of $110 \mathrm{MPa}$.

These results indicate that the transverse tensile strength increases as the area fraction of the matrix occupied on the fracture surface increases. That is, fiber-distribution which makes the cracks propagate throughout the matrix is effective in improving the transverse tensile strength.

\section{Conclusions}

The preform of continuous alumina fibers with fine alumina particles attached to the fiber surfaces was infiltrated with molten $\mathrm{AC} 1 \mathrm{~B}$ aluminum alloy by squeeze casting, and hybrid composites were fabricated. Their microstructure and tensile properties in the range from room temperature to high temperature were compared with those of particle-free composites, and the effects of the particle-dispersion were investigated. The following results were obtained.

(1) In the particle-free composite, many fibers contacted each other, and the fibers were distributed nonuniformly. In the hybrid composite, in which the particles were introduced, the fibers were distributed uniformly because the particles prevented a direct contact between neighboring fibers.

(2) The tensile strength and $0.2 \%$ proof stress, and elastic modulus in the longitudinal direction increased as $V_{\mathrm{f}}$ increased, and these values decreased little up to $623 \mathrm{~K}$. The tensile strength of the hybrid composite was larger than that of the particle-free composite at every temperature. This is because the uniform distribution of the fibers and the reduction in the fiber-to-fiber contact reduce the stress concentration at contact points, and stress transmission between the fiber and the matrix becomes easy.

(3) The tensile strength and the $0.2 \%$ proof stress in the transverse direction decreased as $V_{\mathrm{f}}$ increased. These were improved by the introduction of the particles, leading to the fact that the strength of the hybrid composite was close to that of the unreinforced alloy even at high-temperature. This is because the uniform distribution of the fibers reduces the fracture initiated at the points of fiber contact, and the strong fiber-matrix bond promotes the fracture throughout the matrix.

\section{REFERENCES}

1) H. R. Shetty and T. W. Chou: Metall. Trans. A 16A (1985) 853-864.

2) H. Mabuchi and Y. Nakayama: J. JILM 38 (1988) 608-613.

3) Y. Kagawa and H. C. Bae: J. Japan Inst. Metals 53 (1989) 339-348.

4) K. Matsuura, C. Meikon and N. Matsuda: J. Japan Inst. Metals 54 (1990) 671-678.

5) Y. Ikeuchi, K. Iga and T. Hanabusa: J. Soc. Mater. Sci. JP. 43 (1994) 792-798.

6) K. Yamada, S. Sekiguchi, T. Hanamura and T. Inoue: J. Japan Inst. Metals 59 (1995) 1108-1117.

7) H. N. Liu and K. Ogi: Mater. Sci. Technol. 15 (1999) 821-826.

8) T. Sato, J. Furukawa and K. Shimakage: J. JILM 40 (1990) 822-827.

9) K. T. V. Rao, S. C. Siu and R. O. Ritchie: Metall. Mater. Trans. A 24A 
(1993) 721-734.

10) G. Chadwick: ECCM 4 (1990) 3-13.

11) E. Nakata: in New Materials Handbook, (Maruzen, Tokyo, 1988) pp. $129-140$

12) Y. Nishida: Materia Japan 36 (1997) 40-46.

13) S. De Bondt, L. Froyen and A. Deruyttere: Mater. Sci. Eng. A135 (1991) 29-32.

14) S. Towata, H. Ikuno and S. Yamada: Trans. JIM 29 (1988) 314-321.

15) H. Ikuno, S. Towata, S. Yamada and Y. Awano: J. JILM 44 (1994)
379-384.

16) K. Matsunaga, S. Ochiai, K. Osamura, Y. Waku and T. Yamamura: J. JILM 43 (1993) 219-224.

17) Sumitomo Chemical Co.: Data sheet for Altex (1995).

18) Y. Abe and M. Nakatani: J. JILM 36 (1986) 372-380.

19) M. Morita: in New Materials Handbook, (Maruzen, Tokyo, 1988) pp. 99-113.

20) A. Kelly and N. H. Macmillan: Strong Solids, 3rd (Oxford Univ. Press, 1986) pp. $240-325$. 